\title{
Comparative study of the discrete velocity and lattice Boltzmann methods for rarefied gas flows through irregular channels
}

\author{
Wei Su, ${ }^{1}$ Scott Lindsay, ${ }^{2}$ Haihu Liu, ${ }^{3}$ and Lei $\mathrm{Wu}^{2}$, * \\ ${ }^{1}$ Istituto di Nanotecnologia, Consiglio Nazionale delle Ricerche, 70126, Bari, Italy \\ ${ }^{2}$ James Weir Fluids Laboratory, Department of Mechanical and Aerospace Engineering, \\ University of Strathclyde, Glasgow G1 1 XJ, UK \\ ${ }^{3}$ School of Energy and Power Engineering, Xi'an Jiaotong University, 28 West Xianning Road, Xi'an 710049, China
}

\begin{abstract}
Rooted from the gas kinetics, the lattice Boltzmann method (LBM) is a powerful tool in modeling hydrodynamics. In the past decade, it has been extended to simulate rarefied gas flows beyond the Navier-Stokes level, either by using the high-order Gauss-Hermite quadrature, or by introducing the relaxation time that is a function of the gas-wall distance. While the former method, with a limited number of discrete velocities (e.g. D2Q36), is accurate up to the early transition flow regime, the latter method (especially the multiple relaxation time (MRT) LBM), with the same discrete velocities as that used in simulating hydrodynamics (i.e. D2Q9), is accurate up to the free-molecular flow regime in the planar Poiseuille flow. This is quite astonishing in the sense that less discrete velocities are more accurate. In this paper, by solving the Bhatnagar-Gross-Krook kinetic equation accurately via the discrete velocity method, we find that the high-order GaussHermite quadrature can not describe the large variation in the velocity distribution function when the rarefaction effect is strong, but the MRT-LBM can capture the flow velocity well because it is equivalent to solving the Navier-Stokes equations with an effective shear viscosity. Since the MRTLBM has only been validated in simple channel flows, and for complex geometries it is difficult to find the effective viscosity, it is necessary to assess its performance for the simulation of rarefied gas flows. Our numerical simulations based on the accurate discrete velocity method suggest that the accuracy of the MRT-LBM is reduced significantly in the simulation of rarefied gas flows through the rough surface and porous media. Our simulation results could serve as benchmarking cases for future development of the LBM for modeling and simulation of rarefied gas flows in complex geometries.
\end{abstract}

\section{INTRODUCTION}

When the mean free path $\lambda$ of gas molecules is comparable to or even larger than the characteristic flow length $H$, the traditional Navier-Stokes (NS) equations derived from the continuum-fluid hypothesis fail, and the gas kinetic theory is adopted to describe rarefied gas dynamics. The Boltzmann equation is fundamental to describe the dilute gas dynamics using the velocity distribution function (VDF) of gaseous molecules. According to the Chapman-Enskog expansion, NS equations are only the first-order approximation in the Knudsen number $(\mathrm{Kn}=\lambda / H)$ to the Boltzmann equation [1]. Therefore, they are valid in the continuum flow regime where $\mathrm{Kn} \lesssim 0.001$ [2]. As the Knudsen number increases, higher-order (non-equilibrium) terms begin to dominate, and NS equations gradually loss validity. The non-equilibrium effects not only cause velocity slip and temperature jump at solid surfaces in the slip flow regime $(0.001 \lesssim \mathrm{Kn} \lesssim 0.1)$, but also modify the constitutive relations in the transition $(0.1 \lesssim \mathrm{Kn} \lesssim 10)$ and freemolecular $(10 \lesssim \mathrm{Kn})$ flow regimes such that the Newton's law for stress and strain and Fourier's law for heat flux and temperature gradient do not hold anymore. This leads to a number of counterintuitive phenomena, including the thermal transpiration where gas molecules

* Correspoding author: lei.wu.100@strath.ac.uk along solid surface move towards the hotter region [3], the Knudsen paradox where the dimensionless mass flow rate in Poiseuille flow could increase when the gas pressure decreases 4 , the temperature bimodality in the forcedriven Poiseuille flow [5], the inverted velocity in cylindrical Couette flow [6], and the gas anti-resonance where the shear stress in an oscillating lid-driven cavity flow could be smaller than that of the one-dimensional (1D) Couette flow [7.

In the past decades, due to the rapid development of microelectromechanical systems 8 and the shale gas revolution in North America 9, extensive works have been devoted to construct efficient numerical schemes to simulate gas flows at the micro scale, where the flow velocity is usually far smaller than the most probable speed of the gas molecules. In most of these applications, gas flows vary from the slip to the free-molecular flow regimes and the gas-surface interaction dominates flow behavior. High-fidelity numerical methods to solve the Boltzmann equation include the numerical kernel method [10, the conservative projection-interpolation method [11, the low-variance direct simulation Monte Carlo method [12, and the fast spectral method [13, to name just a few. Due to the high computational cost, however, the Boltzmann equation is usually simplified by the BhatnagarGross-Krook (BGK) equation under the single relaxation time (SRT) approximation [14, which is often solved by the discrete velocity method (DVM) where the continuous molecular velocity space is represented by a few 
number of discrete velocities 15 19. Generally speaking, rarefied gas flows with large values of $\mathrm{Kn}$ need a larger number of discrete velocities to resolve the large variations/discontinuities in the VDF, for instance see the numerical examples in Refs. [13, 20.

Rooted from the gas kinetics, the lattice Boltzmann method (LBM) is a popular and powerful tool in modeling the NS hydrodynamics and beyond. Historically, the SRT-LBM is firstly developed as an alternative solver for the NS equations. Since it uses a very limited but highly optimized number of discrete velocities, e.g. the D2Q9 scheme for 2D problems, the SRT-LBM can be viewed as a special type of DVM to solve the BGK equation [21. To capture the non-equilibrium effects beyond the NS hydrodynamics, a rigorous procedure for obtaining high-order approximations to the BGK equation is proposed 22 . and tested in various canonical problems [23 29]. The basic idea is that, since non-equilibrium effects are related to high-order moments of the VDF, a higher-order quadrature must be used in the simulation of rarefied gas flows. By expanding the VDF into high-order GaussHermite polynomials or Gauss quadrature in the spherical coordinate system, it is found that, with a not very large number of discrete lattice velocities, e.g. D2Q36 or D2Q64, high-order LBM schemes can accurately describe rarefied gas flows up to the early transition flow regime, i.e. $K n \lesssim 0.5$.

In addition to high-order quadrature, Zhang et al. 30. attempted to capture the Knudsen layer structure in rarefied gas flows within the framework of the standard SRTLBM usually for simulation of hydrodynamics, but by introducing the gas kinetic boundary condition and the effective relaxation time that is a function of the gas-wall distance. In the pressure-driven flow, this "wall-scaling" approach provides a significant improvement for Knudsen numbers up to 0.5. Later, this scheme has also been applied to study the rarefied thermal [31] and oscillatory Couette flow 32 in the early transition flow regime with great success.

Further improvement has been made by Guo, Zheng and Shi [33, who developed a numerical scheme within the framework of multiple relaxation time (MRT) LBM. In addition to the "wall-scaling" of the relaxation time, the combined bounce-back and specular-reflection boundary condition is designed, such that the new scheme is equivalent to solving the NS equations with the second-order velocity slip boundary condition, at least in the slip flow regime. In the Poiseuille flow between two parallel plates, it is found that this MRT-LBM scheme is, surprisingly, even able to predict the velocity profile and mass flow rate with good accuracy, up to the freemolecular flow regime. For this reason, the MRT-LBM has attracted significant attentions 33 37.

In the simulation of rarefied gas flows, it is quite astonishing that the MRT-LBM of Guo et al. with fewer number of discrete velocities [33] are more accurate than the LBM of higher-order quadrature 22, 29. Although Guo et al. honestly and explicitly pointed out that their scheme is only designed and analyzed for plane walls 33. and is hard to be extended to generalized gas-surface boundaries 38, it has been widely applied to study the rarefied gas flows through the microchannel with rough surface and complex porous media [36, 37. However, whether this scheme works on complex geometries or not is not clear. Thus, it is the aim of this paper to address the accuracy of the MRT-LBM in simulating rarefied gas flows in complex geometries.

The rest of the paper is organized as follows. In Sec. II] the BGK equation and its numerical scheme is introduced. The accuracy of the DVM is assessed, and the reason why higher-order LBM fails is demonstrated by scrutinizing the structure of the VDF. In Sec. III. by looking at the macroscopic flow velocity and the NS equation with the effective shear viscosity, the reason why the MRT-LBM with modified relaxation times works in planar channels is explained. In Sec. IV, the numerical results of the DVM is compared to those of MRT-LBM for the Couette flow through rough microchannels [36, while in Sec. V the Poiseuille flow through porous media is studied and the accuracy of the regularized MRTLBM [37] is assessed. In Sec. VI the DVM is used to calculate rarefied gas flows around square cylinders in a microchannel to provide benchmarking data for the future development of LBM in simulation of complex microflows. Finally, conclusions are given in Sec. VII.

\section{THE GAS KINETIC THEORY AND THE DVM}

The fundamental Boltzmann equation describes the evolution of the VDF in dependence of spatial position, molecular velocity and time. In this paper, we use the following BGK equation [14] instead of the full Boltzmann equation, in the Cartesian coordinates:

$$
\frac{\partial f}{\partial t}+\mathbf{v} \cdot \frac{\partial f}{\partial \mathbf{x}}+\mathbf{a} \cdot \frac{\partial f}{\partial \mathbf{v}}=\frac{\sqrt{\pi}}{2 \mathrm{Kn}}\left(F_{e q}-f\right) .
$$

Here, $\mathbf{v}=\left(v_{1}, v_{2}, v_{3}\right)$ is the molecular velocity normalized by the most probable speed $v_{m}=\sqrt{2 R T_{0}}$ at the reference temperature $T_{0}$, where $R$ is the gas constant; $\mathbf{x}=\left(x_{1}, x_{2}, x_{3}\right)$ is the spatial coordinate normalized by the characteristic flow length $H ; \mathbf{a}=\left(a_{1}, a_{2}, a_{3}\right)$ is the external acceleration normalized by $v_{m}^{2} / H ; t$ is the time normalized by $H / v_{m} ; f(t, \mathbf{x}, \mathbf{v})$ is the VDF normalized by $n_{0} / v_{m}^{3}$, where $n_{0}$ is the average number density of gas molecules; and $F_{e q}$ is the equilibrium VDF defined as

$$
F_{e q}=\frac{n}{(\pi T)^{3 / 2}} \exp \left(-\frac{|\mathbf{v}-\mathbf{u}|^{2}}{T}\right),
$$

where $n$ is the number density of gas molecules normalized by $n_{0}, T$ is the temperature normalized by $T_{0}$, $\mathbf{u}=\left(u_{1}, u_{2}, u_{3}\right)$ is the macroscopic flow velocity normalized by $v_{m}$. Finally, the Knudsen number is defined as

$$
\mathrm{Kn}=\frac{\lambda}{H},
$$


where the mean free path of gas molecules is related to its shear viscosity $\mu$ of the gas as

$$
\lambda=\frac{\mu\left(T_{0}\right)}{\bar{p}} \sqrt{\frac{\pi R T_{0}}{2}},
$$

with $\bar{p}$ being the average gas pressure at the reference temperature.

Macroscopic gas quantities are obtained from the velocity moments of the VDF. The number density, flow velocity, and temperature are calculated as follows:

$$
\begin{array}{r}
n=\int f d \mathbf{v}, \\
\mathbf{u}=\frac{1}{n} \int \mathbf{v} f d \mathbf{v}, \\
T=\frac{2}{3} \int|\mathbf{v}-\mathbf{u}|^{2} f d \mathbf{v} .
\end{array}
$$

Equation (1) has to be supplied with the gas kinetic boundary condition which determines the VDF of the reflected gas molecules at the surface in terms of that of the incident molecules. In this paper, the Maxwell diffuse-specular boundary condition is used [39]. Suppose the wall has a temperature of $T_{0}$ and moves with the velocity $\mathbf{u}_{w}$, the VDF for the reflected molecules at the wall (i.e. when $\left(\mathbf{v}-\mathbf{u}_{w}\right) \cdot \mathbf{n}>0, \mathbf{n}$ is the outward unit normal vector at the solid surface) is given by

$$
\begin{aligned}
f(\mathbf{x}, \mathbf{v})= & \alpha \frac{n_{w}}{\pi^{3 / 2}} \exp \left(-\left|\mathbf{v}-\mathbf{u}_{w}\right|^{2}\right) \\
& +(1-\alpha) f\left(\mathbf{x}, \mathbf{v}-2\left[\left(\mathbf{v}-\mathbf{u}_{w}\right) \cdot \mathbf{n}\right] \mathbf{n}\right)
\end{aligned}
$$

where $\alpha$ is the tangential momentum accommodation coefficient. The cases of $\alpha=1$ and 0 correspond to the diffusive-reflection and specular-reflection conditions, respectively. A brief review related to the tangential momentum accommodation coefficient can be found in [40]. Finally, $n_{w}$ is defined by

$$
n_{w}=2 \sqrt{\pi} \int_{\left(\mathbf{v}^{\prime}-\mathbf{u}_{w}\right) \cdot \mathbf{n}<0}\left|\left(\mathbf{v}^{\prime}-\mathbf{u}_{w}\right) \cdot \mathbf{n}\right| f\left(\mathbf{x}, \mathbf{v}^{\prime}\right) \mathrm{d} \mathbf{v}^{\prime},
$$

such that the mass flux across the wall is equal to zero.

\section{A. The linearized BGK equation}

When the flow velocity is very small compared to $v_{m}$, and the external acceleration is small, we can linearize the VDF about the global equilibrium state $f_{e q}$ as follows:

$$
f=f_{e q}(1+h), \quad f_{e q}=\frac{\exp \left(-|\mathbf{v}|^{2}\right)}{\pi^{3 / 2}},
$$

and the perturbation $\operatorname{VDF} h(t, \mathbf{x}, \mathbf{v})$ is governed by the following linearized BGK equation [41, 42]:

$$
\begin{array}{r}
\frac{\partial h}{\partial t}+\mathbf{v} \cdot \frac{\partial h}{\partial \mathbf{x}}-2 \mathbf{a} \cdot \mathbf{v}=\mathcal{L}(\varrho, \mathbf{u}, \tau)-\delta h, \\
\mathcal{L}(\varrho, \mathbf{u}, \tau)=\delta\left[\varrho+2 \mathbf{u} \cdot \mathbf{v}+\tau\left(|\mathbf{v}|^{2}-\frac{3}{2}\right)\right],
\end{array}
$$

where $\varrho=\int h f_{e q} \mathrm{~d} \mathbf{v}$ is the perturbed number density, $\mathbf{u}=$ $\int \mathbf{v} h f_{e q} \mathrm{~d} \mathbf{v}$, and $\tau=\frac{2}{3} \int|\mathbf{v}|^{2} h f_{e q} \mathrm{~d} \mathbf{v}-\varrho$ is the perturbed temperature, and the rarefaction parameter is

$$
\delta=\frac{\sqrt{\pi}}{2 \mathrm{Kn}} .
$$

The linearized boundary condition will be given for each specific case considered below.

\section{B. Numerical scheme for general $2 \mathrm{D}$ problems}

The VDF in the linearized BGK equation (11) is defined in a six-dimensional phase space. For general $2 \mathrm{D}$ problems (i.e. in the $x_{1}-x_{2}$ physical space), however, the phase space can be cast into a four-dimensional one, which will reduce the computational cost significantly. This is achieved by introducing the following two reduced VDFs:

$$
\begin{aligned}
& \Phi\left(x_{1}, x_{2}, v_{1}, v_{2}\right)=\frac{1}{\sqrt{\pi}} \int \exp \left(-v_{3}^{2}\right) h d v_{3} \\
& \Psi\left(x_{1}, x_{2}, v_{1}, v_{2}\right)=\frac{1}{\sqrt{\pi}} \int \exp \left(-v_{3}^{2}\right)\left(v_{3}^{2}-\frac{1}{2}\right) h d v_{3} .
\end{aligned}
$$

Note that we are interested in the steady-state solution, so the derivative with respect to the time is omitted in Eq. (11), and hence $\Phi$ and $\Psi$ are time independent. In the following paper, for convenience, we use $\mathbf{v}_{2 D}=\left(v_{1}, v_{2}\right), \mathbf{x}=\left(x_{1}, x_{2}\right), \mathbf{a}=\left(a_{1}, a_{2}\right)$, and $\mathbf{u}=\left(u_{1}, u_{2}\right)$. The macroscopic quantities can now be calculated as $\varrho=\int \Phi f_{e q, 2 D} \mathrm{~d} \mathbf{v}_{2 D}, \mathbf{u}=\int \mathbf{v}_{2 D} \Phi f_{e q, 2 D} \mathrm{~d} \mathbf{v}_{2 D}$, and $\tau=\frac{2}{3} \int\left[\left(\left|\mathbf{v}_{2 D}\right|^{2}-1\right) \Phi+\Psi\right] f_{e q, 2 D} \mathrm{~d} \mathbf{v}_{2 D}$, where the equilibrium state is now given by $f_{e q, 2 D}=\exp \left(-\mathbf{v}_{2 D}^{2}\right) / \pi$.

Multiplying the time-independent linearized BGK equation by $\exp \left(-v_{3}^{2}\right) / \sqrt{\pi}$ and $\exp \left(-v_{3}^{2}\right)\left(v_{3}^{2}-1 / 2\right) / \sqrt{\pi}$, respectively, and integrating the resulting equations with respect to $v_{3}$, we finally obtain the control equations for $\Phi$ and $\Psi$ :

$$
\begin{aligned}
& \mathbf{v}_{2 D} \cdot \frac{\partial \Phi}{\partial \mathbf{x}}=\mathcal{L}_{\Phi}(\varrho, \mathbf{u}, \tau)-\delta \Phi, \\
& \mathbf{v}_{2 D} \cdot \frac{\partial \Psi}{\partial \mathbf{x}}=\mathcal{L}_{\Psi}(\tau)-\delta \Psi
\end{aligned}
$$

where $\mathcal{L}_{\Phi}(\varrho, \mathbf{u}, \tau)=\delta\left[\varrho+2 \mathbf{u} \cdot \mathbf{v}_{2 D}+\tau\left(\left|\mathbf{v}_{2 D}\right|^{2}-1\right)\right]+$ $2 \mathbf{a} \cdot \mathbf{v}_{2 D}$ and $\mathcal{L}_{\Psi}(\tau)=\delta \tau / 2$.

For numerical simulations of Eqs. (14) and (15), the 2D molecular velocity space $\mathbf{v}$ is represented by discrete velocities. To capture the discontinuities/rapid variations in the VDF at large $\mathrm{Kn}, v_{1}$ and $v_{2}$ are represented by $N_{v}$ non-uniform points in each direction [13]:

$$
v_{1,2}=\frac{4}{\left(N_{v}-1\right)^{3}}\left(-N_{v}+1,-N_{v}+3, \cdots, N_{v}-1\right)^{3},
$$

where the discrete velocities are distributed in a square of dimension $[-4,4]^{2}$, and are refined near $v_{1,2}=0$. 


\begin{tabular}{c|c|c|c|c|c}
\hline \hline$\delta$ & Cercignani & DVM32 & GH8 & GH32 & GH64 \\
\hline 0.01 & 3.0499 & 3.0522 & 1.0877 & 1.4743 & 1.6658 \\
0.05 & 2.3026 & 2.3022 & 1.0991 & 1.4783 & 1.6554 \\
0.1 & 2.0313 & 2.0327 & 1.1131 & 1.4820 & 1.6415 \\
0.5 & 1.6025 & 1.6018 & 1.2131 & 1.4878 & 1.5509 \\
1 & 1.5396 & 1.5386 & 1.3162 & 1.4977 & 1.5212 \\
5 & 1.9928 & 1.9903 & 1.9421 & 1.9813 & 1.9861 \\
10 & 2.7669 & 2.7674 & 2.7322 & 2.7608 & 2.7643 \\
\hline \hline
\end{tabular}

TABLE I. Dimensionless mass flow rates in the Poiseuille flow between two parallel plates. The data in the column 'Cercignani' are obtained from Tabel I and II in Ref. 43, while those of 'DVM32', 'GH8', 'GH32', and 'GH64' are the DVM results when the velocity space is discretized by Eq. (16) with $N_{v}=32$, and Gauss-Hermite quadrature with 8, 32, and 64 abscissas, respectively.

Note that the molecular velocity space can also be discretized according to the Gauss-Hermite quadrature, but this type of discretization is not accurate at large Knudsen numbers, as will be demonstrated below.

Equations (14) and (15) are solved by the following iterative method:

$$
\begin{aligned}
& \delta \Phi^{(j+1)}+\mathbf{v}_{2 D} \cdot \frac{\partial \Phi^{(j+1)}}{\partial \mathbf{x}}=\mathcal{L}_{\Phi}\left(\varrho^{(j)}, \mathbf{u}^{(j)}, \tau^{(j)}\right), \\
& \delta \Psi^{(j+1)}+\mathbf{v}_{2 D} \cdot \frac{\partial \Psi^{(j+1)}}{\partial \mathbf{x}}=\mathcal{L}_{\Psi}\left(\tau^{(j)}\right)
\end{aligned}
$$

where the superscripts $(j)$ and $(j+1)$ represent two consecutive iteration steps, and the spatial derivatives are approximated by the second-order upwind finite difference. The iteration is terminated when the relative error in macroscopic flow velocity between two consecutive iteration steps is less than $10^{-5}$.

\section{Poiseuille flow between two parallel plates}

To verify the accuracy of our numerical scheme, we calculate the Poiseuille flow between two infinite parallel plates, and compare the numerical results with those obtained by Cercignani and Daneri [43], where the BGK equation with the diffuse boundary condition is reduced to a purely integral one analytically, which is then solved by the discrete ordinate method numerically.

Consider two stationary parallel plates with a distance of $H$. After the spatial normalization, they locate at $x_{2}=-1 / 2$ and $x_{2}=1 / 2$, respectively. The gas flow between the two plates is driven by a pressure gradient along the $x_{1}$ direction. As long as the pressure gradient is small, the governing equation for the perturbed VDF can be given by Eq. (14), and the dimensionless mass flow rate

$$
Q=\int_{-1 / 2}^{1 / 2} u_{1}\left(x_{2}\right) d x_{2}
$$
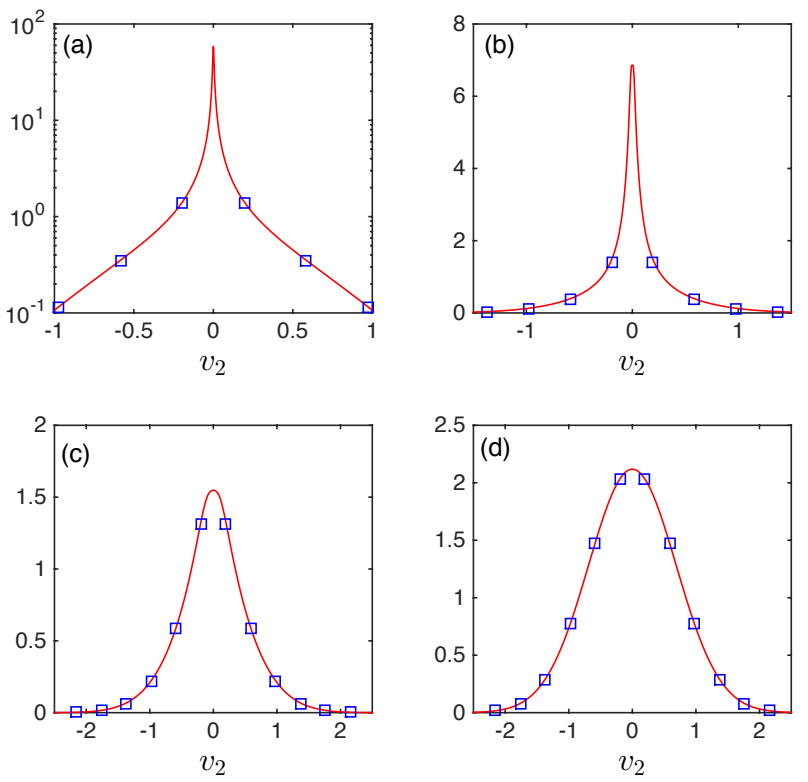

FIG. 1. The marginal VDF $\int v_{1} \Phi\left(x_{2}=0, \mathbf{v}_{2 D}\right) f_{e q, 2 D} d v_{1}$ in the force-driven Poiseuille flow between two parallel plates. The rarefaction parameter $\delta$ in the linearized BGK equation is (a) $\delta=0.01$, (b) $\delta=0.1$, (c) $\delta=1$, and (d) $\delta=10$. Solid lines: the marginal VDF obtained from DVM with $N_{v}=320$ in Eq. 16. Note that $N_{v}=32$ is enough to get accurate results, we use $N_{v}=320$ here just to show the detailed structure of the VDF. Squares: results from the high-order Gauss-Hermite quadrature with 32 abscissas in each velocity direction.

can be found by setting $\mathbf{a}=(1,0)$. From Eq. (11) we know that the VDF has the symmetry $h\left(v_{1}\right)=-h\left(-v_{1}\right)$, so according to the diffuse boundary condition (9) and the linearization (10), the reflected VDFs $\Phi$ and $\Psi$ at the two walls are zero. Since this problem is effectively $1 \mathrm{D}$, the spatial derivative with respect to $x_{1}$ is omitted, while the spatial domain $-1 / 2 \leq x_{2} \leq 1 / 2$ is discretized by 100 uniform cells.

Table II compares the mass flow rate obtained from the DVM of different velocity discretization schemes with those of Cercignani and Daneri 43 . It is clearly seen that the DVM has very good accuracy when the molecular velocity space is discretized by Eq. 16 with $N_{v}=32$, where the relative error is within $0.13 \%$. On the contrary, when the molecular velocity space is discretized by the Gauss-Hermite quadrature, the accuracy of the DVM is only good at large values of $\delta$ (or equivalently, small values of $\mathrm{Kn}$ ) when the flow is in the slip regime; at small values of $\delta$ (i.e. transition and free molecular flow regimes), the DVM with Gauss-Hermite quadrature yields wrong results even when there are 64 discretized velocities in each velocity direction. For example, when $\delta=0.01$, the volume mass flow rate is underestimated by $50 \%$. Further increase of the number of lattice velocities could increase the simulation accuracy, but the convergence to the exact solution is rather slow.

The fundamental reason behind the unsatisfied performance of the Gauss-Hermite quadrature is that, as $\delta$ de- 
creases, gas molecules in the force-driven Poiseuille flow concentrate near $v_{2}=0$, and the VDF varies rapidly near this region, see Fig. 1(a). In this case, the minimum value of the discretized velocities is $v_{2}=0.1948$ in the 'GH32' scheme (see the definition in Table I) cannot reflect the large variation of the $\mathrm{VDF}$ in the region of $\left|v_{2}\right| \lesssim 0.05$. On the contrary, when $v_{2}$ is discretized by Eq. 116 with $N_{v}=32$, we have $\min \left(\left|v_{2}\right|\right)=0.00013$ and there are 8 discrete velocities in the region of $\left|v_{2}\right|<0.05$; therefore, the large variation of the VDF is captured and the mass flow rate is almost the same as that obtained from the "semi-analytical" method [43. In the following simulations, if without specification, we use the non-uniform velocity discretizations 16 with $N_{v}=32$.

It is worth mentioning that the accuracy of the DVM has also been verified by the direct simulation Monte Carlo method of Bird 44, for rarefied gas flows through porous media; for example, see the details in the Fig. 3 of Ref. 45] and the corresponding texts.

\section{HOW DOES THE MRT-LBM WORK IN PLANAR CHANNEL?}

For the discussion in the above Sec. II C we know that, in order to capture the physical phenomena at the mesoscopic level, that is, to resolve the VDF, we need a reasonably large amount of discrete velocities; and once the VDF is obtained accurately, the macroscopic flow quantities are also accurately captured. The MRT-LBM, with only 9 discrete velocities, certainly cannot describe the large variation of the VDF in Fig. 1(a) and (b). However, various numerical simulations have shown that the MRT-LBM with modified relaxation times can capture the flow velocity in the planar Poiseuille flow [33, 34, up to the free-molecular flow regime. Thus, to understand how the MRT-LBM works, we need to look at the macroscopic level.

To this end, we first plot the typical velocity profiles of Poiseuille flow in Fig. 2(a). It is clear that, when $\delta$ is large (or equivalently the Knudsen number is small), the gas has nearly the same slip velocities at the wall. When $\delta$ decreases, the slip velocity increases. Meanwhile, the velocity profile becomes flatter. The competition between the two effects leads to the Knudsen minimum at $K n \sim 1$. That is, the dimensionless mass flow rate is minimum, see also the data in Table I

We then look at the governing equation for the flow velocity $u_{1}$. According to the Chapman-Enskog expansion [1], for the specific problem in Sec. II C, the equation in the dimensionless form can be written as follows:

$$
\frac{\partial}{\partial x_{2}}\left(\operatorname{Kn} \frac{\partial u_{1}}{\partial x_{2}}+\text { High-order terms }\right)=-\sqrt{\pi},
$$

where the high-order terms are contributions beyond the hydrodynamic level; details form of which are very complicated (for example see those in Burnett equation [46]) and is not given here. Note that the shear viscosity of the
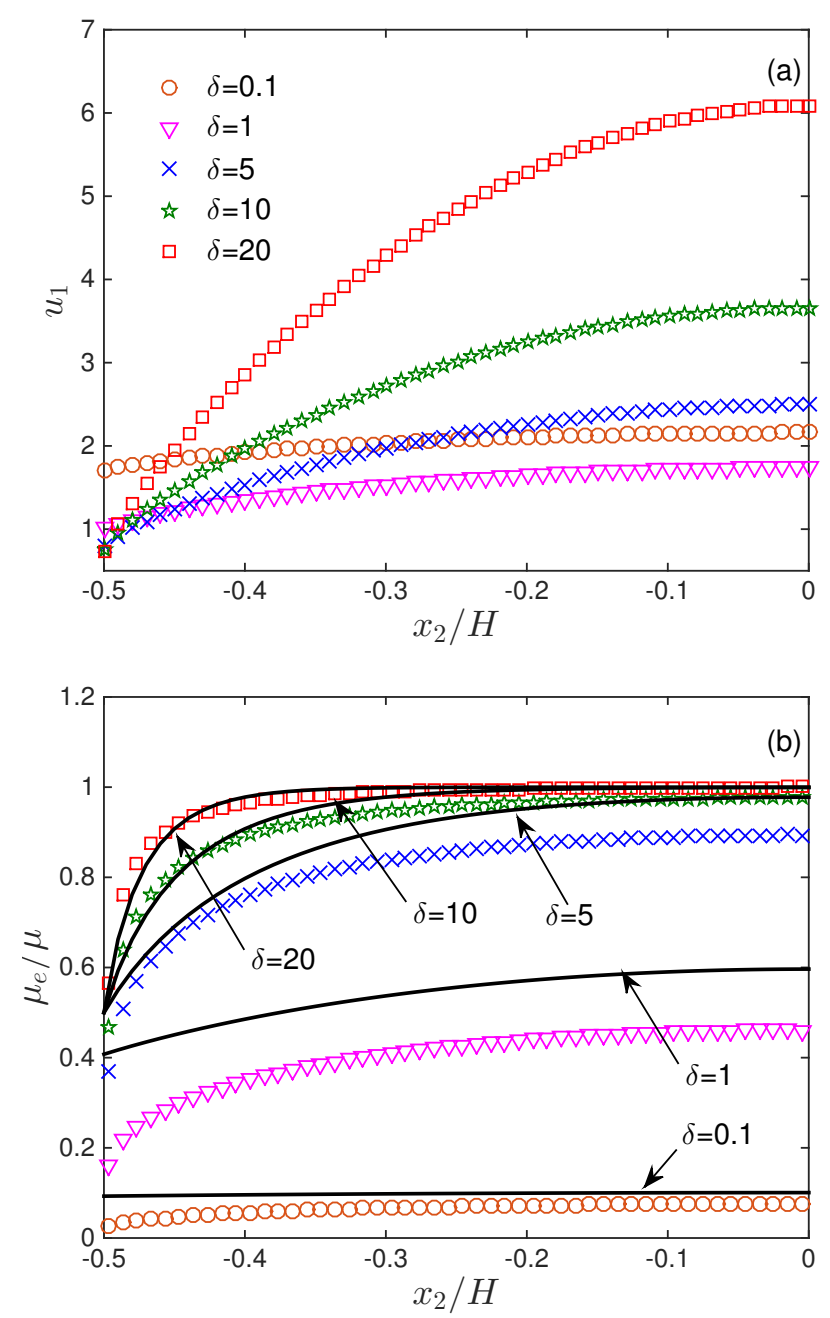

FIG. 2. (a) The velocity profile in the Poiseuille flow between two parallel plates, obtained from the DVM with $N_{v}=32$ in Eq. 16. For symmetry, only half of the spatial domain is shown. (b) The ratio between the effective viscosity and real viscosity of gas. Symbols are the numerical results according to Eq. 25]. Solid lines are the analytical results given by Eq. 22. Also note that according to Eq. 23, $\mu_{e} / \mu$ is 0.9186 , $0.8494,0.7383,0.3607$, and 0.0534 when $\delta$ is $20,10,5,1$, and 0.1 , respectively.

gas has been implicitly included in the Knudsen number according to Eqs. (3) and (4), and the Knudsen number is spatial-independent.

An easy way to take the high-order terms into account is to introduce the effective viscosity $\mu_{e}$ and approximate Eq. 19 by the following form:

$$
\frac{\partial}{\partial x_{2}}\left(\operatorname{Kn}_{e} \frac{\partial u_{1}}{\partial x_{2}}\right)=-\sqrt{\pi}
$$

where $\mathrm{Kn}_{e}$ is the effective Knudsen number; it is related to the effective shear viscosity as

$$
\frac{\mathrm{Kn}_{e}}{\mathrm{Kn}}=\frac{\mu_{e}}{\mu}
$$


and $\mu_{e}$ could dependent on the spatial variable or not.

From the simple kinetic theory we know that the shear viscosity is proportional to the mean free path of gas molecules [1. It is also argued by some authors that the mean free path near the solid wall is reduced as compared to that in the bulk region. So for flows between two parallel plates, Guo et al. introduced the following spatial-dependent effective shear viscosity [33]:

$$
\frac{\mu_{e}}{\mu}=\frac{1}{2}\left[\psi\left(\frac{2 x_{2}+1}{2 \mathrm{Kn}}\right)+\psi\left(\frac{1-2 x_{2}}{2 \mathrm{Kn}}\right)\right],
$$

where $\psi(z)=1+(z-1) \exp (-z)-z^{2} \int_{1}^{\infty} t^{-1} \exp (-z t) d t$, while Li et al. introduced the spatial-independent correction to the shear viscosity as [34]:

$$
\frac{\mu_{e}}{\mu}=\frac{1}{1+2 \mathrm{Kn}}
$$

Note that there are many other forms of the effective shear viscosity in the literature. We only introduce these two forms here because they have been implemented in the MRT-LBM.

The approximate equation (20) with the effective shear viscosity (22) does explain the rigorous solution from the BGK equation to some extent. Integrating Eq. 20 with respect to $x_{2}$ and considering the symmetry condition at $x_{2}=0$, we have

$$
\mathrm{Kn}_{e} \frac{\partial u_{1}}{\partial x_{2}}=-\sqrt{\pi} x_{2}
$$

which leads to

$$
\frac{\mu_{e}}{\mu}=\frac{\mathrm{Kn}_{e}}{\mathrm{Kn}}=-2 \delta\left(\frac{\partial u_{1}}{\partial x_{2}}\right)^{-1}
$$

With the DVM solution to the BGK equation (11), we can obtain the ratio between the effective and real shear viscosities numerically using Eq. (25). We compare it to the analytical expression 22 and show the results in Fig. 2(b). Clearly, when $\delta=20$ and 10, the agreement between the numerical and analytical solutions of $\mu_{e} / \mu$ is good. This explains why the MRT-LBM, with appropriate boundary conditions, gives excellent velocity profiles of Poiseuille flow with those from the direct simulation Monte Carlo method, see Fig. 7 in Ref. 33. As $\delta$ decreases, the analytical expression of Guo et al. is always higher than the numerical results; specifically, large differences between the two are observed near the Knudsen minimum where $\delta \sim 1$ (or Kn $\sim 1$ ). This explains why the velocity profiles obtained from the MRTLBM of Guo et al. have the largest discrepancy with the results from the direct simulation Monte Carlo method, when the Knudsen number is around one (see Fig. 7 in Ref. [33]).

This deficiency of the MRT-LBM has been reduced by Li et al., who adopted the effective shear viscosity (23). From the caption of Fig. 2 we can see that, this special

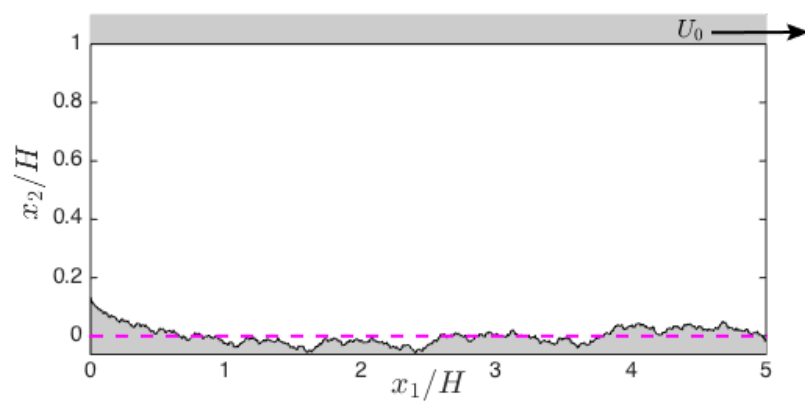

FIG. 3. The geometry of the rough channel as used in Couette flow. Shaded regions are solid plates, while the gas flows in the white region.

form of $\mu_{e} / \mu$ has value roughly equal to the spatial average value of $\mu_{e} / \mu$ from the numerical solution of the BGK equation. For instances, when $\delta=1, \mu_{e} / \mu$ is 0.3607 from Eq. (23), while the numerical value of $\mu_{e} / \mu$ varies from 0.2 near the wall to 0.47 in the middle of the channel; when $\delta=5, \mu_{e} / \mu$ is 0.7383 from Eq. (23), while the numerical value of $\mu_{e} / \mu$ varies from 0.4 near the wall to 0.89 in the middle of the channel. Therefore, the results of the MRT-LBM with the effective shear viscosity (23) agree well with the solutions of the direct simulation Monte Carlo method, see Fig. 1 in Ref. 34].

From the above discussion and comparison, together with the fact that the MRT-LBM uses a special technique to recover the second-order velocity slip boundary condition of Cercignani [47, we may conclude that the MRT-LBM with effective relaxation times 33,34 is an alternative solver for the approximate Navier-Stokes equation 20 with the effective shear viscosity. Therefore, the accuracy of this method depends strongly on how accurate the effective shear viscosity is. The effective viscosities given by Eqs. (22) and (23) have only been validated in simple channels, whether they work in complex geometries or not has not been rigorously assessed. In general, we believe the determination of the effective viscosity like Eqs. (22) and (23) in complicated geometry is difficult, because it is even hard to define the local Knudsen number.

The following sections are devoted to assess the accuracy of the MRT-LBM for rarefied gas flow simulations in complex geometries.

\section{COUETTE FLOW IN ROUGH MICROCHANNELS}

Now we begin to assess the accuracy of MRT-LBM in the simulation of rarefied gas flows in irregular channels, by comparing the numerical results obtained from deterministic solutions of the linearized BGK equation.

As Ref. 36, we first consider the Couette flow between two plates with a distance of $H$, see Fig. 3. The smooth top plate moves in the $x_{1}$ direction with a speed $U_{0}$, while 
the stationary bottom plate has a roughness described by the following Weierstrass-Mandelbrot fractal function:

$$
r\left(x_{1} / H\right)=A \sum_{n=n_{1}}^{\infty} \frac{\cos \left(2 \pi \gamma^{n} x_{1} / H\right)}{\gamma^{(2-D) n}},
$$

where $D$ is the self-affine fractal dimension and $\gamma$ determines the frequency spectrum of the surface roughness.

The aspect ratio of the microchannel (i.e. the length over the height of the channel) is 5 , and we choose $\gamma=1.5$ and $n$ from -4 to 30 in Eq. (26). The scaling parameter $A$ is used to adjust the surface roughness $\epsilon$ :

$$
\epsilon=\frac{\sigma}{H}
$$

where $\sigma$ is the root mean square of the fractal function (26). For instance, for a rough surface of $\epsilon=2 \%$, when $\overline{\mathrm{D}}=1.5$, we choose $A=0.0148$.

At the inlet and outlet of the computational domain, the periodic boundary condition is used, while at the solid surface, the diffuse boundary condition is used 39 . That is, at the moving smooth plate located at $x_{2}=1$, we have

$$
h\left(x_{1}, 1, \mathbf{v}\right)=2 \sqrt{\pi} \int_{v_{2}^{\prime}>0} v_{2}^{\prime} h\left(x_{1}, 1, \mathbf{v}^{\prime}\right) f_{e q}\left(\mathbf{v}^{\prime}\right) \mathrm{d} \mathbf{v}^{\prime}+\frac{2 U_{0}}{v_{m}} v_{1},
$$

while at the bottom rough plate located,

$$
h(\mathbf{x}, \mathbf{v})=2 \sqrt{\pi} \int_{\mathbf{v}_{n}^{\prime} \cdot \mathbf{n}<0}\left|\mathbf{v}_{n}^{\prime} \cdot \mathbf{n}\right| h\left(\mathbf{x}, \mathbf{v}^{\prime}\right) f_{e q}\left(\mathbf{v}^{\prime}\right) \mathrm{d} \mathbf{v}^{\prime} .
$$

The boundary conditions for the two reduced VDFs $\Phi$ and $\Psi$ can be obtained with the help of Eq. (13).

Note that the Weierstrass-Mandelbrot fractal surface is not differentiable, therefore, the rough surface is approximated by the "stair case" in the numerical simulation, as used in the MRT-LBM simulation [36. Also, we are interested in the horizontal velocity profile of the gas and the mass flow rate at different level of gas rarefaction, when the steady state is reached. We take $U_{0} / v_{m}=1$ in Eq. 287, so the horizontal flow velocity is calculated as

$$
u_{1}\left(x_{1}, x_{2}\right)=U_{0} \int v_{1} \Phi\left(x_{1}, x_{2}, \mathbf{v}_{2 D}\right) \mathrm{d} \mathbf{v}_{2 D},
$$

while the average mass flow rate, which is normalized by $\bar{p} H U_{0} / R T_{0}$, is given by

$$
Q=\frac{\iiint v_{1} \Phi\left(x_{1}, x_{2}, \mathbf{v}_{2 D}\right) \mathrm{d} \mathbf{v}_{2 D} \mathrm{~d} x_{1} \mathrm{~d} x_{2}}{5},
$$

where the denominate 5 equals to the normalized length of the channel in the horizontal direction, as shown in Fig. 3 .

In all the numerical calculations, the $2 \mathrm{D}$ molecular velocity space $\mathbf{v}_{2 D}$ is discretized by $32 \times 32$ non-uniform points. The physical space is also discretized into Cartesian grids. In the $x_{1}$ direction, 1500 equidistant points
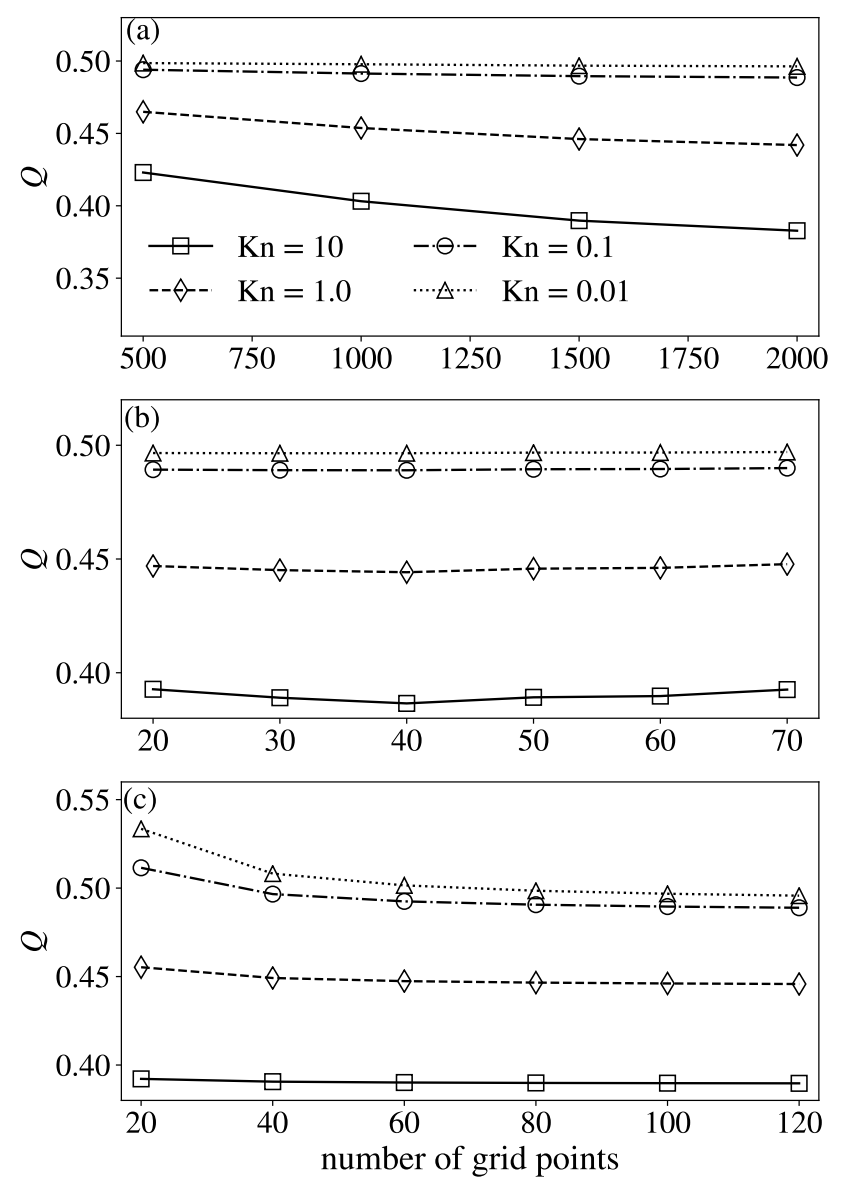

FIG. 4. The convergence test of the mass flow rate $Q$ obtained from the DVM, for Couette flow in a rough channel of $\epsilon=2 \%$ and $D=1.5$ : The influence of the number of grid points in (a) the $x_{1}$ direction, (b) the $x_{2}$ direction within the rough region, that is, $x_{2} \in[\min (r), \max (r)]$, and (c) the $x_{2}$ direction outside the rough region, that is, $x_{2} \in(\max (r), 1]$.

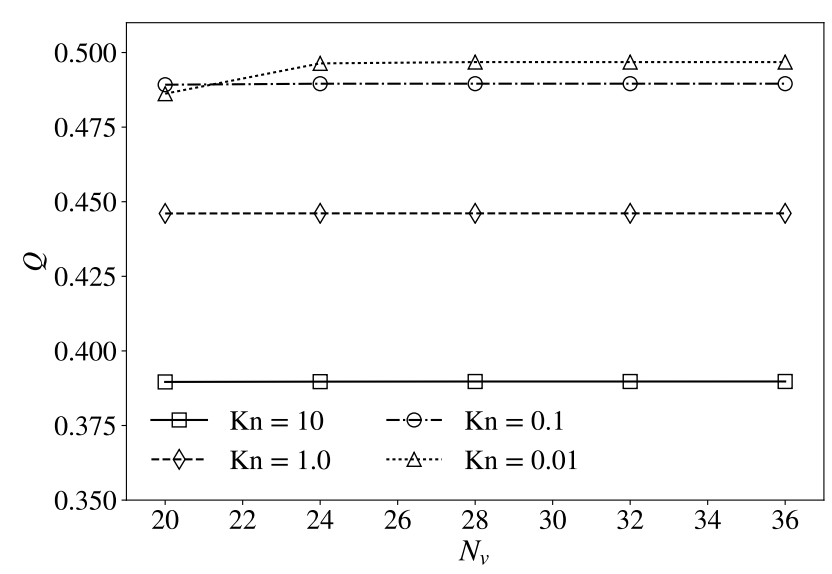

FIG. 5. The convergence test of the mass flow rate $Q$ obtained from the DVM, for the Couette flow in a rough channel of $\epsilon=2 \%$ and $D=1.5$ : the influence of the number of discrete velocity points when Eq. 16 is used. 

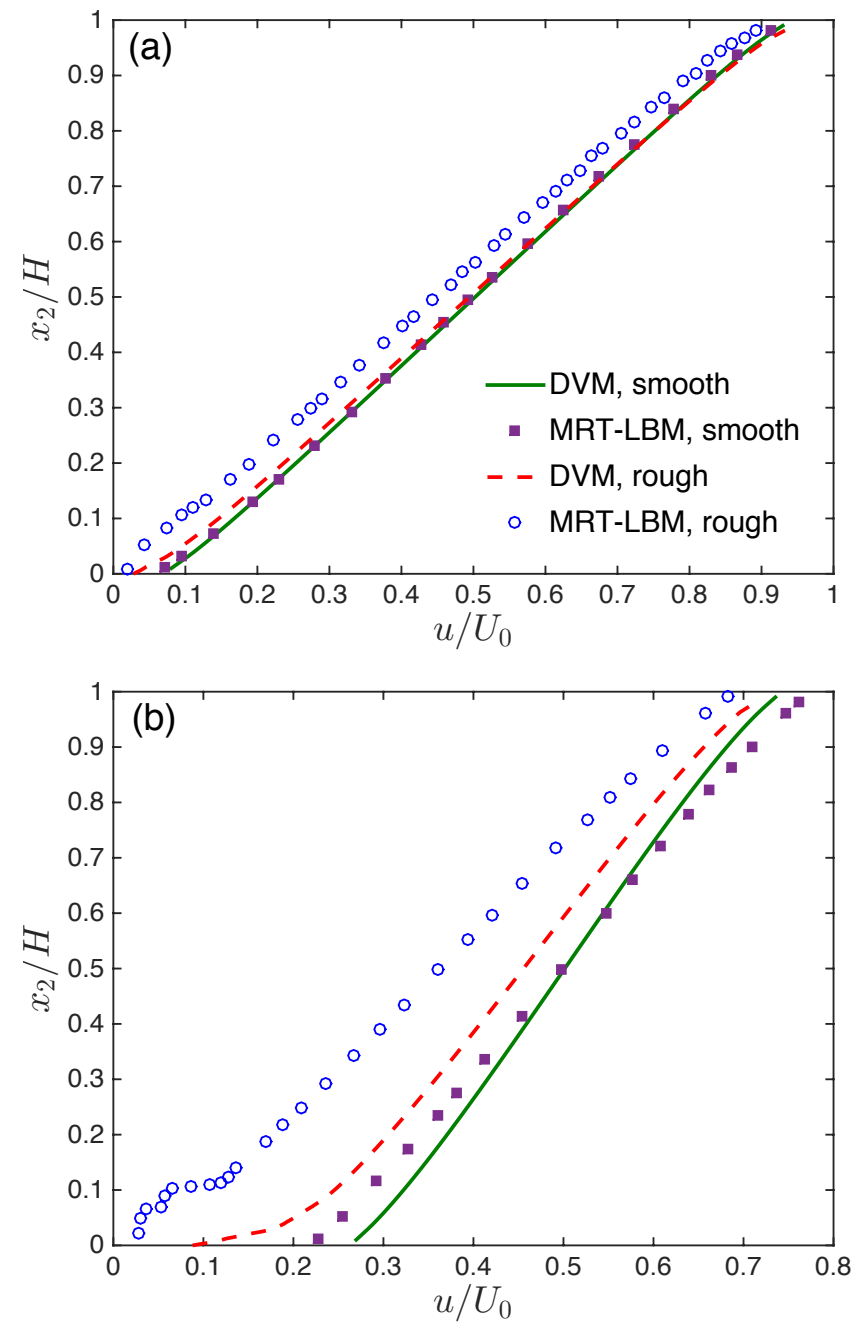

FIG. 6. Comparisons in the average streamwise velocity profile of the Couette flow in microchannels between the DVM and MRT-LBM [36], when $D=1.5, \epsilon=2 \%$, (a) $\mathrm{Kn}=0.1$ and (b) $\mathrm{Kn}=1$. "Smooth" here means that both plates are smooth, while "rough" means that only the bottom plate has a rough surface, see Fig. 3

are used. In the $x_{2}$ direction, the rough region $\min (r) \leq$ $x_{2}<\max (r)$ is discretized by 60 equidistant points, while the rest is discretized by 100 equidistant points. Convergence studies were performed, and it has been found in Figs. 4 and 5 that further refinement of the velocity and spatial grids would only improve the solutions (i.e. mass flow rates in the rough channel of $\epsilon=2 \%$ and $D=1.5$, under 4 selected Kn numbers) by a magnitude no more than $2 \%$.

We first compare the horizontal velocity profiles between the numerical results obtained from the DVM and MRT-LBM with the "wall-scaling" of the relaxation time, when $\mathrm{Kn}=0.1$ and 1 . When both plates are smooth, from Fig. 6 we find that MRT-LBM is accurate at $\mathrm{Kn}=0.1$. However, at $\mathrm{Kn}=1$, the slip velocity at the bottom surface from the MRT-LBM simulation is

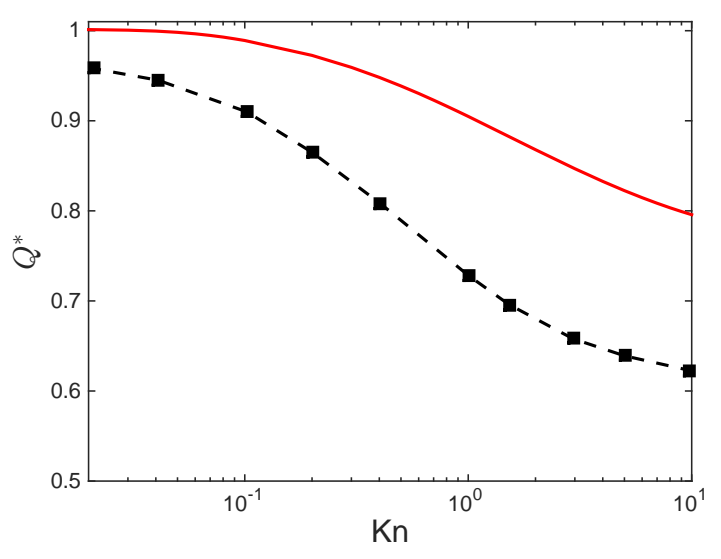

FIG. 7. The ratio between the mass flow rates in the rough ( $D=1.5$ and $\epsilon=2 \%)$ and smooth channels. Solid line: DVM. Squares: MRT-LBM from [36].

about $15 \%$ smaller than that from the DVM. Nevertheless, the mass flow rates from both numerical schemes are quite close, as the MRT-LBM predicts a larger velocity close to the top plate. When the bottom surface is rough, the accuracy of the MTR-LBM is reduced significantly. For example, at $\mathrm{Kn}=1$ and $x_{2}=0.1 H$ (i.e. the rough region), the average horizontal flow velocity from the DVM simulation is two and a half times larger than that from the MRT-LBM. Possible reasons for the failure of MRT-LBM include (i) the combined bounce-back and specular-reflection boundary condition is only derived in a large flat plate, which may not work in a rough surface, and (ii) for non-plane surface, the "wall-scaling" of the relaxation time may not work properly. Actually, it has been observed that, the extend NS equations incorporating a wall-scaling model cannot give a correct prediction in velocity profiles even for a simple circular cylinder flow when $\mathrm{Kn}>0.4$ [38.

We then consider the influence of the surface roughness on the mass flow rate. As Ref. [36], we study how the following ratio

$$
Q^{*}=\frac{Q_{r}}{Q_{s}},
$$

change with the Knudsen number. Here $Q_{r}$ and $Q_{s}$ are the average mass flow rates through the channels with rough and smooth surfaces, respectively. For the smooth channel with diffuse boundary condition, our numerical simulations show that $Q_{s}$ is always 0.5 in the whole flow regime. This can actually be proven in the continuum and slip flow regimes using the NS equations with the first-order velocity slip boundary condition, and in the free-molecular flow regime using the gas kinetic theory. The numerical results for the fractal surface with $D=1.5$ and $\epsilon=2 \%$ are shown in Fig. 7. Clearly, the accuracy of MRT-LBM decreases as the Knudsen number increases. At $\mathrm{Kn}=10, Q^{*}$ obtained from MRT-LBM is about $25 \%$ smaller than that from the DVM. This error, however, 
is in fact very large by taking into account the fact that the value of $Q^{*}$ only decreases by $20 \%$ from the continuum flow regime to the free-molecular flow regime with $\mathrm{Kn}=10$. In this sense, the MRT-LBM overestimates the decrease of mass flow rate $Q^{*}$ by twice.

We have also investigated the mass flow rate when the fractal dimension $D$ in Eq. (26) takes different values. Similar behaviors as that in Figs. 6 and 7 are observed (not shown). These examples show that the MRT-LBM is not reliable in simulating Couette flow in rough microchannels.

\section{POISEUILLE FLOW IN POROUS MEDIA}

In this section, we further access the accuracy of the MRT-LBM in simulating rarefied Poiseuille flows through three porous media (Medium 1 with irregular solids; Medium 2 with square solids; Medium 3 with circular solids), as shown in Fig. 8. The vertical size of each porous medium is assumed to be $H$. The investigation of the rarefied gas flows in porous media becomes popular due to the shale gas revolution in North America, and LBM is widely used to calculate the apparent permeability (a parameter describing how fast the gas can be extracted) of porous media. We will study the variation of the apparent permeability with respect to the Knudsen number using the DVM, and access the accuracy of MRT-LBM with the "wall-scaling" of the relaxation time used in Ref. 37.

The DVM to solve the linearized BGK equation for this problem can be found in Ref. [45, where the periodic boundary condition in the horizontal direction is used. We have also tested that the pressure boundary condition adopted in Ref. 37] leads to the same value of apparent gas permeability as long as the pressure difference is very small.

According to Ref. [45, the apparent gas permeability $k_{a}$, which is normalized by $H^{2}$, is calculated by

$$
k_{a}=\sqrt{\frac{4}{\pi}} Q \mathrm{Kn},
$$

where $Q$ is the dimensionless mass flow rate.

Since different porous medium has different distribution of pore radius, it will be useful to define the characteristic length as

$$
H^{*}=H \sqrt{\frac{12 k_{\infty}}{\phi}},
$$

where $\phi$ is the porosity of the porous medium and $k_{\infty}$ is the intrinsic permeability, i.e. the apparent permeability when $\mathrm{Kn} \rightarrow 0$. Hence the equivalent Knudsen number is defined as

$$
\mathrm{Kn}^{*}=\frac{\lambda}{H^{*}}=\mathrm{Kn} \frac{H}{H^{*}}=\mathrm{Kn} \sqrt{\frac{\phi}{12 k_{\infty}}} .
$$
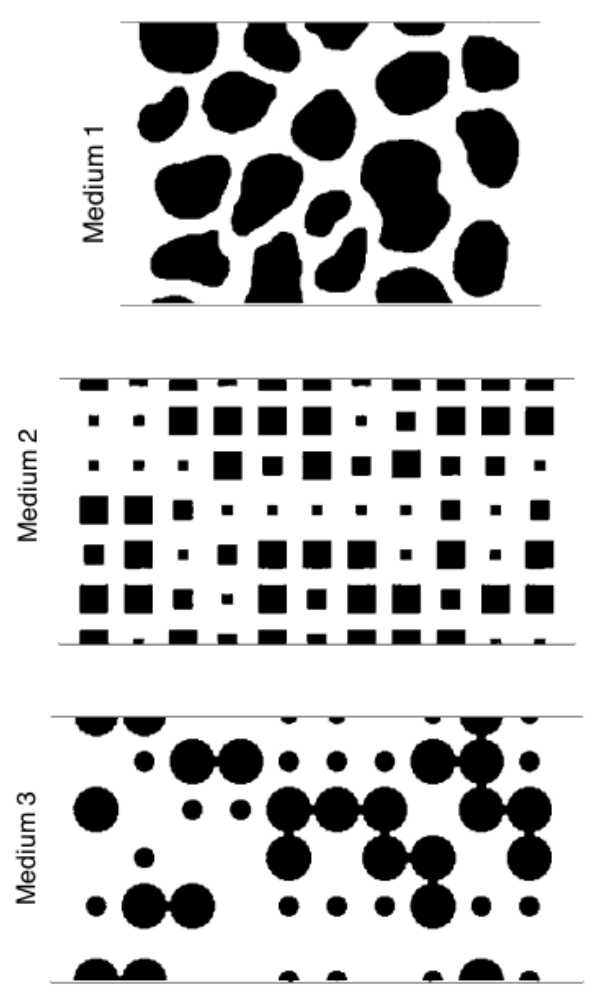

FIG. 8. Different porous media used in the numerical simulation 37. The gas flows from left to right.

The comparison on the apparent gas permeability of the three porous media at different $K n^{*}$ between the numerical results from the DVM and MRT-LBM is presented in Fig. 9. Our DVM simulations show that, for the porous media 1 and 3 , the ratio of the apparent gas permeability to the intrinsic permeability, i.e. $k_{a} / k_{\infty}$, is nearly a linear function of the Knudsen number $\mathrm{Kn}^{*}$ : the results could be described by the same fitting form as

$$
\frac{k_{a}}{k_{\infty}}=1+c \mathrm{Kn}^{*}
$$

where $c=4.7$ and 5.2 for the media 1 and 3 , respectively.

However, for the porous medium $2, k_{a} / k_{\infty}$ obtained from the DVM is clearly a convex function of $K n^{*}$; in the region considered in Fig. 9, it can be fitted as

$$
\frac{k_{a}}{k_{\infty}}=1+7 \mathrm{Kn}^{*}+0.13\left(\mathrm{Kn}^{*}\right)^{2}
$$

The MRT-LBM results in Fig. 9 are obtained from Ref. [37, where the authors presented a few data points for the three types of porous media for $\mathrm{Kn}^{*}$ ranging from 0.01 to 6.26. Then these MRT-LBM data were used to verify the accuracy of two commonly used semiempirical models for estimation of apparent permeability [48, 49]. It is worth noting that these empirical analytical models are derived for Poiseuille flow through plane plates and/or cylindrical capillaries instead of the complex porous media, based on the Navier-Stokes equations 


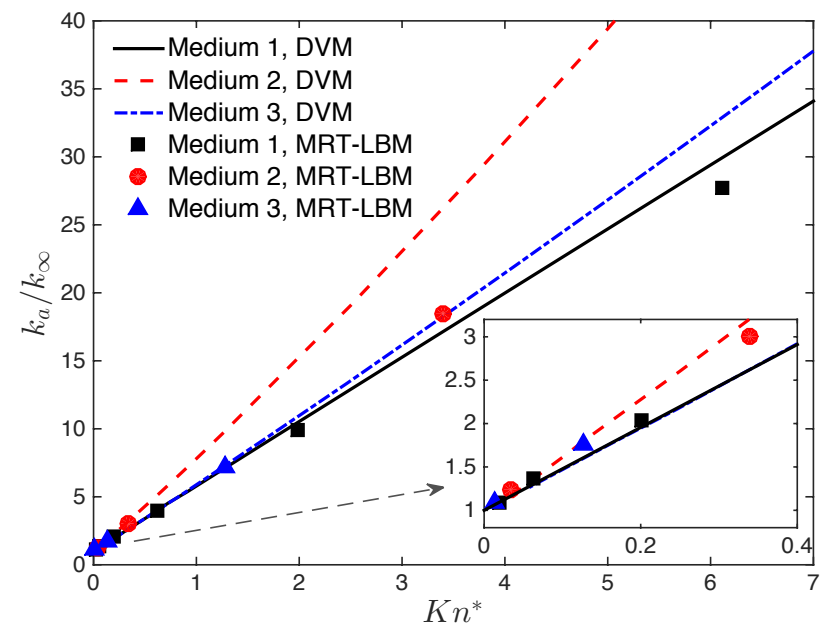

FIG. 9. The ratio of the apparent gas permeability $k_{a}$ to the intrinsic permeability $k_{\infty}$ as a function of the equivalent Knudsen number $\mathrm{Kn}^{*}$. Results of MRT-LBM simulations are obtained from Ref. 37.

with first-order velocity slip boundary condition or the kinetic theory. From the comparison in Fig. 9, we find that 1) for the three porous media, both DVM and MRTLBM results agree well with each other in slip and early transitional flow regimes, and 2) as the Knudsen number increases, notable discrepancies are observed between DVM and MRT-LBM results, e.g. the apparent permeability from DVM for medium 1 is larger than that from MRT-LBM by about $10 \%$ at $\mathrm{Kn}^{*} \simeq 6.26$; even worse, the apparent permeability predicted from the MRT-LBM at $\mathrm{Kn}^{*} \simeq 3.45$ is nearly half of that from the DVM. Note that there is no available data to assess the performance of the MRT-LBM for medium 3 at large Kn numbers; since there are only a few MRT-LBM data points, it is hard to estimate an upper bound of the Knudsen number, above which the MRT-LBM might become problematic in estimating the apparent gas permeability.

In general, the MTR-LBM might not describe the variation of the apparent gas permeability with respect to the shape and configuration of pores in porous media at large Knudsen numbers, and should not viewed as an accurate numerical method to simulate rarefied gas flows in porous media.

\section{POISEUILLE AND COUETTE FLOWS IN SQUARE CYLINDER CHANNEL}

Finally, we consider the flows in a microchannel with a square cylinder placed at the center region, see Fig. 10. Poiseuille flow in such a channel was used to discuss the applicability and limitations of the MRT-LBM for simulating complex flows in the slip-flow regime [50. It is easy to understand that, when one wants to discuss the applicability of a simulation method for engineering problems, a model verification only in canonical flows with simple

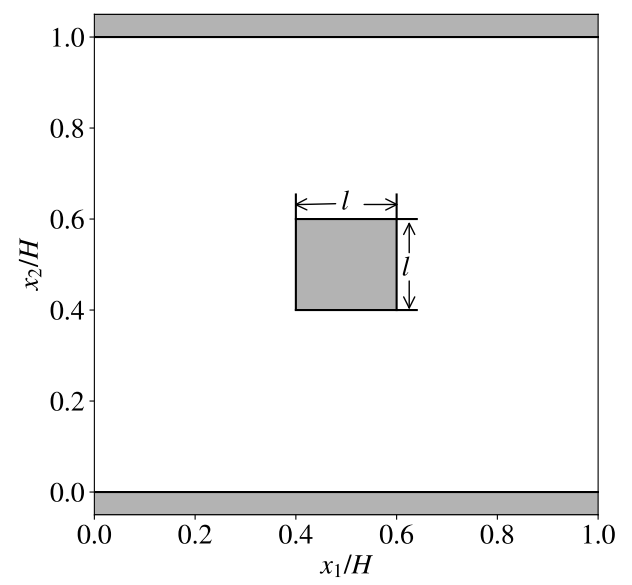

FIG. 10. The geometry for the simulation of gas flows around a square cylinder in a microchannel. Shaded regions are solids. The channel height and width are set as $H$, while the dimensions of cylinder is set as $l \times l$.

geometries is not sufficient. However, as far as we are aware of, there are not enough benchmarking tests of complex microflows in the literature. Thereby, the authors only used the normalized velocity profiles from the molecular dynamics (MD) simulation to verify the MRT$\mathrm{LBM}$ at $\mathrm{Kn} \simeq 0.1$ in Ref. [37, [50]. However, there is a clear deficiency in these comparisons between the MD and MRT-LBM: in the MRT-LBM the diffuse boundary condition for the gas-wall interaction is used, while in MD simulation the gas-wall interaction is determined by the intermolecular potential. Therefore, it is very likely that the gas-wall interaction results a non-unitary tangential momentum accommodation coefficient, as observed in Ref. 51; and the accuracy of the MRT-LBM in simulating rarefied gas flows cannot be justified even when its results agree well with the MD simulation.

In the present paper, based on the DVM simulation of rarefied gas flows around a square cylinder in a microchannel, we intend to provide a set of benchmarking data for a strict test of the MRT-LBM in simulating complex micro/nano-scale gas flows, or for the future development of efficient numerical techniques for rarefied gas flow simulations.

To account for the influence of the gas-wall interaction, we use different values of the tangential momentum accommodation coefficient in Eq. 8). Both the Poiseuille flow driven by a constant acceleration $a_{1}=1$ in the $x_{1}$ direction and Couette flow driven by the top moving plate are considered. The apparent permeability (33) for Poiseuille flow and normalized mass flow rate (31) for Couette flow are obtained under a wide range of $\mathrm{Kn}$. We also consider $l=0.2 \mathrm{H}$ and $0.5 \mathrm{H}$ to study the effect of the square dimension, see Fig. 10. The inlet and outlet boundaries are set as periodic boundary condition and thus the calculated domain is regarded as part of an infinite cylinder array set in a microchannel. For all numerical simulations, 117 grid points are put in both $x_{1}$ 


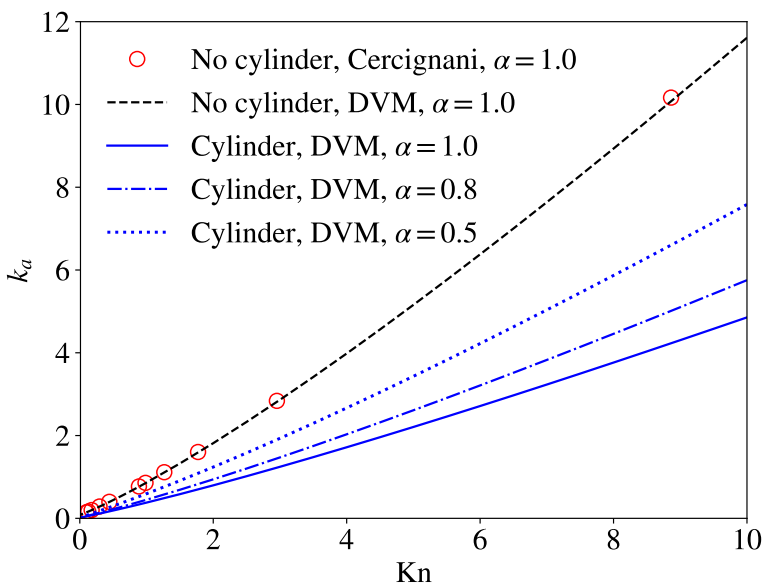

FIG. 11. The variation of the apparent gas permeability of the Poiseuille flow around a square cylinder of dimension $0.2 \mathrm{H} \times 0.2 \mathrm{H}$ in a microchannel, as a function of the Knudsen number, at three different values of the tangential momentum accommodation coefficient. Results indicated by 'Cercignani' are calculated using the dimensionless flow rates by Cercignani and Daneri 43 .

and $x_{2}$ directions with refinement in the vicinity of walls. A convergence study shows that further increasement of the numbers of grid points would improve the solutions by a magnitude no more than $0.5 \%$.

Figure 11 shows the apparent gas permeability of the Poiseuille flow around a square cylinder of dimension $0.2 H \times 0.2 H$ in a microchannel. The $2 \mathrm{D}$ molecular velocity space is discretized by Eq. (16) with $N_{v}=32$, except in the case of $\alpha=0.5$ we choose $N_{v}=64$ at $\mathrm{Kn} \approx 10$. We also include the results for channel flow without the cylinder for reference. It is noted that the appearance of the obstruction reduces the apparent gas permeability $k_{a}$ due to the additional friction. For instance, when $\mathrm{Kn}=\alpha=1, k_{a}$ is decreased from 0.8639 to 0.3801 . As the accommodation coefficient $\alpha$ decreases (more molecules bounce specularly from walls), $k_{a}$ gradually rises due to the increased velocity slip at the wall, see Fig. 12, when $\mathrm{Kn}=1$, we have $k_{a}=0.4497$ and 0.5970 when $\alpha=0.8$ and 0.5 , respectively.

Figure 13 shows the average mass flow rates $Q$ for the Couette flow in the same microchannel. DVM solutions with $N_{v}=64$ are considered as converged, since decreasing $N_{v}$ to 32 only results a change of mass flow rate less than $2.5 \%$. Note that when there is no obstacle between the two parallel plates, the mass flow rate is always 0.5 when the tangential momentum accommodation coefficient is 1 . In the presence of the obstruction, average mass flow rates are reduced by more than $50 \%$. For example, at $\mathrm{Kn}=0.1$ and $\alpha=1, Q$ is equal to 0.2427 for channel flow around a square cylinder. We also plot the horizontal velocity $u_{1}$ along vertical lines at $x_{1}=0$, $x_{1}=0.25$ and $x_{1}=0.5$ in Fig. 14 . We define $\bar{u}_{1, \text { up }}$ and $\bar{u}_{1, \text { lo }}$ as the average horizontal flow velocity within the upper half region $([0.0,1.0] \times[0.5,1.0])$ and lower half
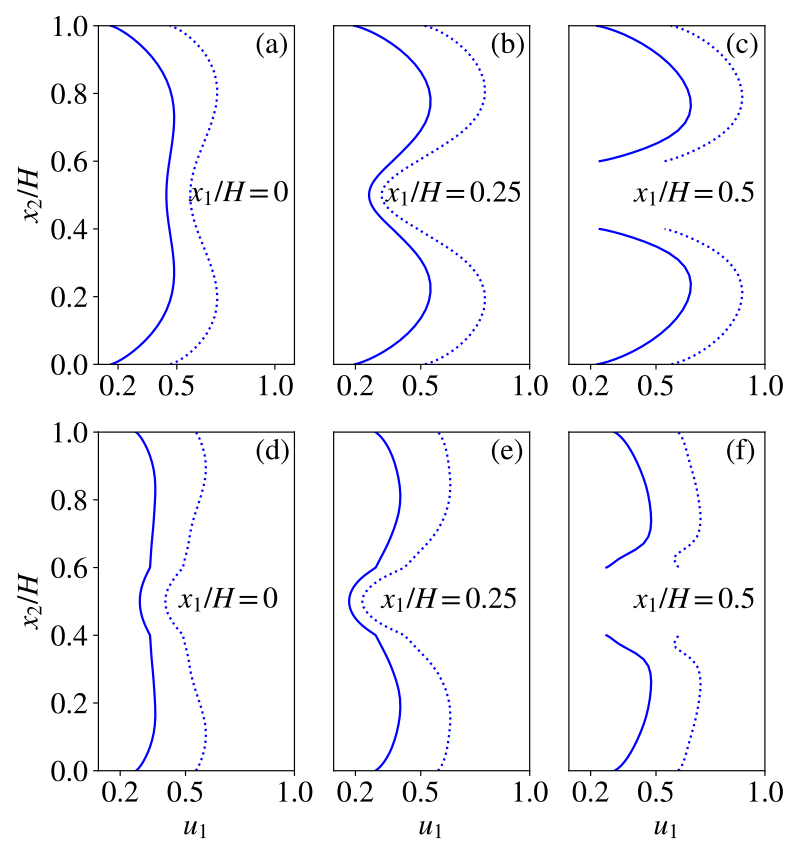

FIG. 12. The horizontal velocity profile in the Poiseuille flow around a square cylinder of dimension $0.2 \mathrm{H} \times 0.2 \mathrm{H}$ in a microchannel: the influence of the Knudsen number and tangential momentum accommodation coefficient $\alpha$. Solid lines: $\alpha=1.0$. Dotted lines: $\alpha=0.5$. The Knudsen numbers in the first and second rows are $K n=0.1$ and 1 , respectively. The velocity profiles for $\alpha=0.8$ are not shown, as they located between those for $\alpha=1$ and $\alpha=0.5$.

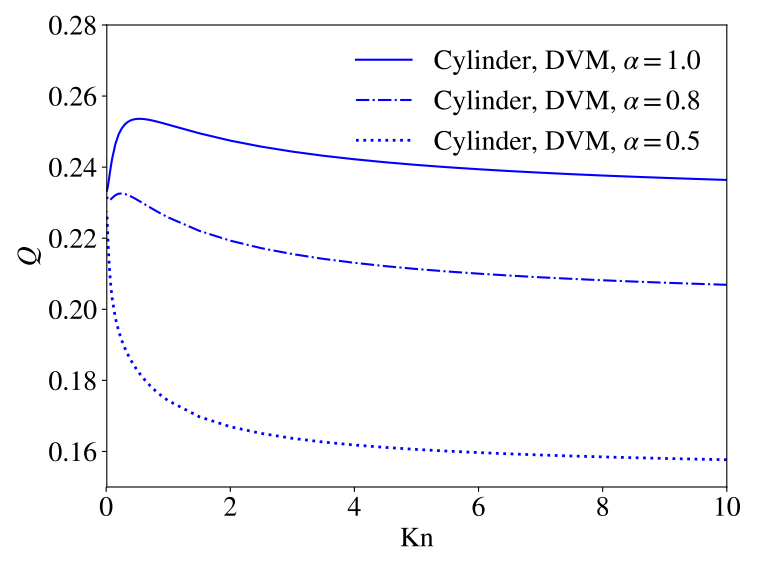

FIG. 13. The mass flow rate of the Couette flow around a square cylinder of dimension $0.2 \mathrm{H} \times 0.2 \mathrm{H}$ in a microchannel.

region $([0.0,1.0] \times[0.0,0.5])$ respectively. Then, further investigation shows that $\bar{u}_{1, \mathrm{up}}=0.3030, \bar{u}_{1, \mathrm{lo}}=0.0326$ for flow around cylinder at $\mathrm{Kn}=0.1$ and $\alpha=1$, while those for flow in an empty channel are $\bar{u}_{1, \text { up }}=0.7128$, $\bar{u}_{1, \mathrm{lo}}=0.2926$. This indicates that the shear driven flow below the cylinder is suppressed significantly. Moreover, as the accommodation coefficient $\alpha$ decreases, $Q$ is further reduced since the velocity-slip near the top plate decreases fast while that near the bottom plate increases 

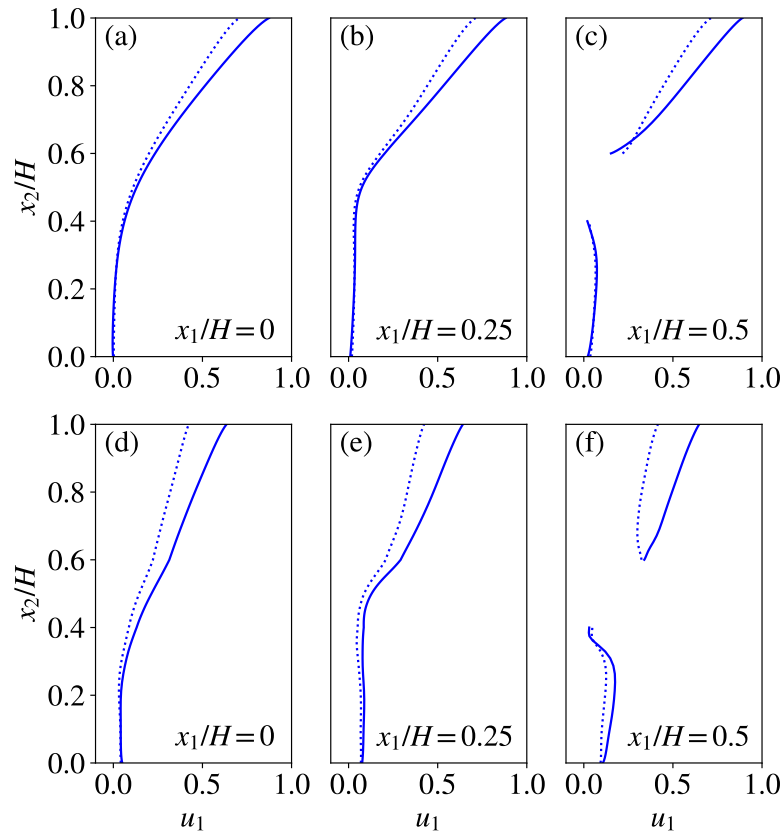

FIG. 14. The horizontal velocity profile in the Couette flow around a square cylinder of dimension $0.2 \mathrm{H} \times 0.2 \mathrm{H}$ in a microchannel. Solid lines: $\alpha=1$. Dotted lines: $\alpha=0.5$. The Knudsen numbers in the first and second rows are $\mathrm{Kn}=0.1$ and 1, respectively. For clarity, the results of $\mathrm{Kn}=0.8$ is not shown, but they lie between the results of $\mathrm{Kn}=1$ and 0.5 .

only a bit. This is in sharp contrast to the Poiseuille flow, where the mass flow rate (or equivalently the apparent permeability) increases when $\alpha$ decreases.

Finally, we plot the apparent permeability in Poiseuille flow and the mass flow rate in Couette flow in Fig. 15. when the size of square cylinder in Fig. 10 is $0.5 H \times 0.5 H$. As expected, they further decreases due to the reduction of flow passage when compared to flows passing a smaller cylinder. For example, when $\alpha=1, k_{a}$ is now equal to 0.1297 when $\mathrm{Kn}=1$, while $Q$ is equal to 0.1299 when $\mathrm{Kn}$ $=0.1$. The effect of the tangential momentum accommodation coefficient on $k_{a}$ and $Q$ is similar as that for cylinder of $0.2 H \times 0.2 H$. It is also interesting to note that the mass flow rates in Couette flow always decrease when Kn increases for the obstruction of dimensions $0.5 \mathrm{H} \times 0.5 \mathrm{H}$, while for the obstruction of dimensions $0.2 \mathrm{H} \times 0.2 \mathrm{H}$, the mass flow rates first increase and then decrease when Kn increases, at $\alpha=1$ and 0.8 , see Fig. 13 .

\section{CONCLUSIONS}

In summary, we have conducted a systematic assessment of the accuracy of the MRT-LBM with a "wallscaling" of the relaxation time in simulating rarefied gas flows in rough microchannels and complex porous media, over a wide range of the Knudsen number. By solving the linearized BGK equation accurately via the DVM, we have shown that:
1. for Couette flow in microchannels, the MRT-LBM can predict the velocity profile with high accuracy in the slip flow regime, when the two surfaces are smooth. However, as the Knudsen number increases or surface roughness emerges, the MRTLBM losses accuracy in predicting the velocity-slip and mass flow rate: it even becomes very inaccurate when the Knudsen number is one.

2. for Poiseuille flow through porous media, the apparent gas permeabilities in complex media with different shape and configuration of pores could not be describe by a single polynomial fitting. The permeabilities predicted by the MRT-LBM agree well with those from the DVM at small Knudsen numbers. However, as the degree of rarefaction increases, large discrepancies emerge between the results from the MRT-LBM and DVM. In one of the case considered, the MRT-LBM underestimates the apparent permeability by nearly twice.

Therefore, for rarefied gas flows through rough surface and complex porous media, the MRT-LBM should not be viewed as an accurate method. Possible reasons for the failure of the MRT-LBM include (i) the combined bounce-back and specular-reflection boundary condition is only derived in a large flat plate, which may not work in an irregular surface, and (ii) for complex geometries, the "wall-scaling" of the relaxation time may not work properly. In our opinion, the MRT-LBM with the "wall-scaling" relaxation time is a numerical technique attempted to capture the rarefaction effects, which can not considered as an appropriate kinetic model to describe the real physical system. This is because, theoretically, if the continuous molecular velocity space is discretized by enough velocity points, one should get accurate results. The original BGK model has been proven accurate when enough appropriate discrete velocities is adopted. However, if the modified BGK model with the "wall-scaling" relaxation time is solved with the same discrete velocities, it will give different solutions, which are incorrect. Nevertheless, it may be still attractive if one can design appropriate boundary conditions and effective relaxation times for general porous media flows in the framework of the MRT-LBM, due to its simplicity and the less-demeaning memory cost.

To this end, the accurate DVM has been used to simulate channel flows around a square cylinder of two different sizes. A set of data on apparent gas permeability for Poiseuille flow and mass flow rates for Couette flow, as well as the velocities profiles, are obtained under a wide range of Knudsen numbers, and the tangential momentum accommodation coefficient in the Maxwell's diffusespecular gas-surface boundary conditions. Our simulation results from the DVM with enough discrete velocity grids could serve as benchmarking cases for future development of the LBM for modeling and simulation of low-speed rarefied gas flows in complex geometry. 

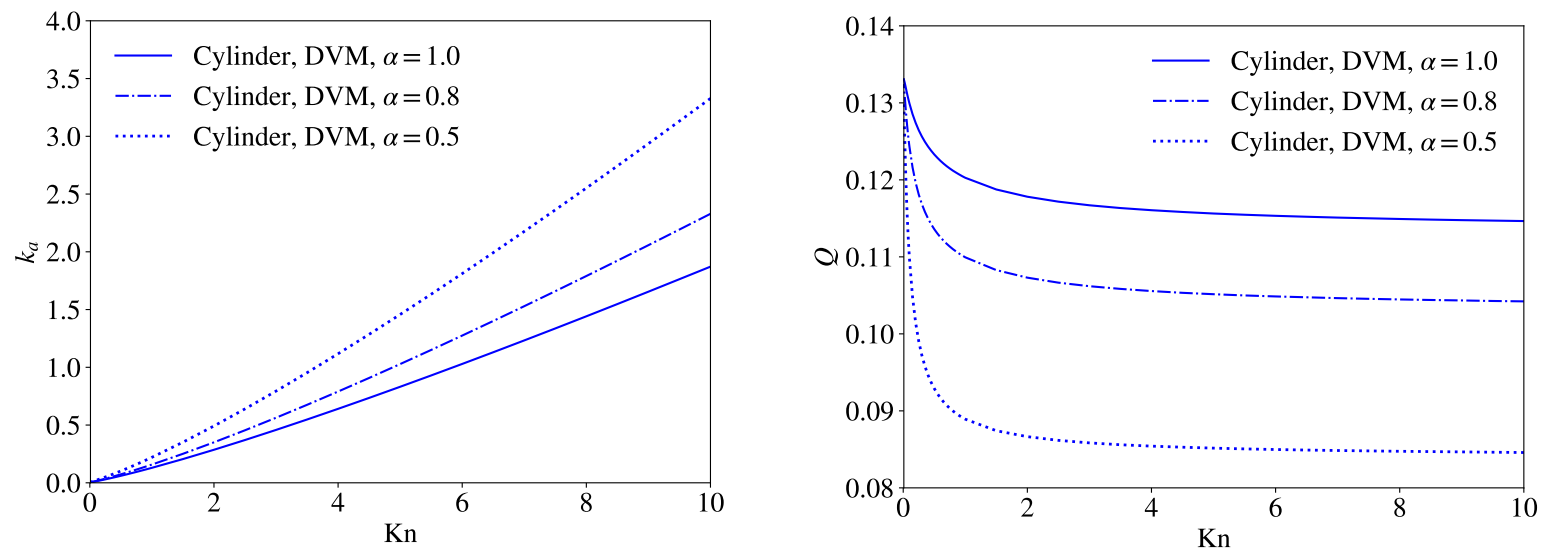

FIG. 15. The apparent gas permeability of the Poiseuille flow (left) and the mass flow rates of the Couette flow (right) around a square cylinder of dimensions $0.5 H \times 0.5 H$ in a microchannel.

L.W. and H.L. acknowledge the financial support of the joint project from the Royal Society of Edinburgh and National Natural Science Foundation of China under grant no. 51711530130. The authors are grateful to the referees for their helpful suggestions.
[1] S. Chapman and T.G. Cowling, The Mathematical Theory of Non-uniform Gases (Cambridge University Press, 1970).

[2] M. Gad-el-Hak, "The fluid mechanics of microdevices the Freeman Scholar lecture," J. Fluids Eng. 121, 5-33 (1999).

[3] O. Reynolds, "On certain dimensional properties of matter in the gaseous state," Phil. Trans. R. Soc. Lond. 170, 727-845 (1879).

[4] W. Steckelmacher, "Knudsen flow 75 years on: the current state of the art for flow of rarefied gases in tubes and systems," Rep. Prog. Phys. 49, 1083-1107 (1999).

[5] M. M. Mansour, F. Baras, and A. L. Garcia, "On the validity of hydrodynamics in plane Poiseuille flows," Physica A 240, 255 (1997).

[6] K. Aoki, H. Yoshida, T. Nakanishi, and A. L. Garcia, "Inverted velocity profile in the cylindrical Couette flow of a rarefied gas," Physical Review E 68, 016302 (2003).

[7] L. Wu, J. M. Reese, and Y. H. Zhang, "Oscillatory rarefied gas flow inside rectangular cavities," J. Fluid Mech. 748, 350-367 (2014).

[8] G. Karniadakis, A. Beskok, and N. Aluru, Microflows and Nanoflows: Fundamentals and Simulation (Springer, 2005).

[9] Q. Wang, X. Chen, A. Jha, and H. Rogers, "Natural gas from shale formation - The evolution, evidences and challenges of shale gas revolution in United States," Renew. Sust. Energ. Rev. 30, 1-28 (2014).

[10] T. Ohwada, Y. Sone, and K Aoki, "Numerical analysis of the Poiseuille and thermal transpiration flows between two parallel plates on the basis of the Boltzmann equation for hard sphere molecules," Phys. Fluids 1, 2042 (1989).

[11] F. G. Tcheremissine, "Direct numerical solution of the Boltzmann equation," AIP Conf. Proc. 762, 677-685
(2005).

[12] T. M. M. Homolle and N. G. Hadjiconstantinou, "A low-variance deviational simulation Monte Carlo for the Boltzmann equation," J. Comput. Phys. 226, 2341-2358 (2007).

[13] L. Wu, J. M. Reese, and Y. H. Zhang, "Solving the Boltzmann equation by the fast spectral method: application to microflows," J. Fluid Mech. 746, 53-84 (2014).

[14] P. L. Bhatnagar, E. P. Gross, and M. Krook, "A model for collision processes in gases. I. Small amplitude processes in charged and neutral one-component systems," Phys. Rev. 94, 511-525 (1954).

[15] C. K. Chu, "Kinetic-theoretic description of the formation of a shock wave," Phys. Fluids 8, 12 (1965).

[16] J. Y. Yang and J. C. Huang, "Rarefied flow computations using nonlinear model Boltzmann equations," J. Comput. Phys. 120, 323-339 (1995).

[17] F. Sharipov, "Rarefied gas flow through a slit. influence of the boundary condition," Phys. Fluids 8, 262 (1996).

[18] K. Xu and J. C. Huang, "A unified gas-kinetic scheme for continuum and rarefied flows," J. Comput. Phys. 229, 7747-7764 (2010).

[19] Z. Guo, K. Xu, and R. J. Wang, "Discrete unified gas kinetic scheme for all Knudsen number flows: Low-speed isothermal case," Phys. Rev. E 88, 033305 (2013).

[20] S. Taguchi and K. Aoki, "Rarefied gas flow around a sharp edge induced by a temperature field," J. Fluid Mech. 694, 191-224 (2012).

[21] X. He and L. S. Luo, "Theory of the lattice Boltzmann method: From the Boltzmann equation to the lattice Boltzmann equation," Phys. Rev. E 56, 6811-6817 (1997).

[22] X. Shan, X. Yuan, and H. Chen, "Kinetic theory representation of hydrodynamics: a way beyond the NavierStokes equation," J. Fluid Mech. 550, 413-441 (2006). 
[23] S. H. Kim, H. Pitsch, and I. D. Boyd, "Accuracy of higher-order lattice Boltzmann methods for microscale flows with finite Knudsen numbers," J. Comput. Phys. 227, 8655-8671 (2008).

[24] J. Meng and Y. H. Zhang, "Accuracy analysis of highorder lattice Boltzmann models for rarefied gas flows," J. Comput. Phys. 230, 835-849 (2011).

[25] J. Meng and Y. H. Zhang, "Gauss-Hermite quadratures and accuracy of lattice Boltzmann models for nonequilibrium gas flows," Phys. Rev. E 83, 036704 (2011).

[26] Y. Shi, P. L. Brookes, Y. W. Yap, and J. E. Sader, "Accuracy of the lattice Boltzmann method for lowspeed noncontinuum flows," Phys. Rev. E 83, 045701(R) (2011).

[27] V. E. Ambruş and V. Sofonea, "High-order thermal lattice Boltzmann models derived by means of Gauss quadrature in the spherical coordinate system," Phys. Rev. E 86, 016708 (2012).

[28] V. E. Ambruş and V. Sofonea, "Implementation of diffuse-reflection boundary conditions using lattice Boltzmann models based on half-space Gauss-Laguerre quadratures," Phys. Rev. E 89, 041301 (2014).

[29] Y. Shi, Y. W. Yap, and J. E. Sader, "Linearized lattice Boltzmann method for micro- and nanoscale flow and heat transfer," Phys. Rev. E 92, 013307 (2015).

[30] Y. H. Zhang, X. J. Gu, R. W. Barber, and D. R. Emerson, "Capturing Knudsen layer phenomena using a lattice Boltzmann model," Phys. Rev. E 74, 046704 (2006).

[31] Y. H. Zhang, X. J. Gu, R. W. Barber, and D. R. Emerson, "Modelling thermal flow in the transition regime using a lattice Boltzmann approach," Europhys. Lett. 77, 30003 (2007).

[32] G. H. Tang, X. J. Gu, R. W. Barber, D. R. Emerson, and Y. H. Zhang, "Lattice Boltzmann simulation of nonequilibrium effects in oscillatory gas flow," Phys. Rev. E 78, 026706 (2008).

[33] Z. Guo, C. Zheng, and B. Shi, "Lattice Boltzmann equation with multiple effective relaxation times for gaseous microscale flow," Phys. Rev. E 77, 036707 (2008).

[34] Q. Li, Y. L. He, G. H. Tang, and W. Q. Tao, "Lattice Boltzmann modeling of microchannel flows in the transition flow regime," Microfluidics Nanofluidics 10, 607-618 (2011).

[35] Y. Yuan and S. Rahman, "Extended application of lattice Boltzmann method to rarefied gas flow in microchannels," Physica A 463, 25-36 (2016).

[36] Z. Deng, Y. Chen, and C. Shao, "Gas flow through rough microchannels in the transition flow regime," Phys. Rev. E 93, 013128 (2016).

[37] J. Zhao, J. Yao, A. Li, M. Zhang, L. Zhang, Y. Yang, and H. Sun, "Simulation of microscale gas flow in heterogeneous porous media based on the lattice Boltzmann method," J. Appl. Phys. 120, 084306 (2016).

[38] Z. Guo, J. Qin, and C. Zheng, "Generalized second-order slip boundary condition for nonequilibrium gas flows," Phys. Rev. E 89, 013021 (2014).

[39] J. C. Maxwell, "On stresses in rarefied gases arising from inequalities of temperature," Philosophical Transactions of the Royal Society Part 1 170, 231-256 (1879).

[40] W. M. Zhang, G. Meng, and X. Y. Wei, "A review on slip models for gas microflows," Microfluid Nanofluid 13, 845-882 (2012).

[41] C. Cercignani, The Boltzmann Equation and Its Applications (Springer, New York, 1988).

[42] F. Sharipov and I. A. Graur, "Rarefied gas flow through a zigzag channel," Vacuum 86, 1778-1782 (2012).

[43] C. Cercignani and A. Daneri, "Flow of a rarefied gas between two parallel plates," J. Appl. Phys. 34, 3509 (1963).

[44] G. A. Bird, Molecular Gas Dynamics and the Direct Simulation of Gas Flows (Oxford Science Publications, Oxford University Press Inc, New York, 1994).

[45] L. Wu, M. T. Ho, L. Germanou, X. J. Gu, C. Liu, K. Xu, and Y. H. Zhang, "On the apparent permeability of porous media in rarefied gas flows," J. Fluid Mech. 822, 398-417 (2017).

[46] L. S. Garcia-Colin, R. M. Velasco, and F. J. Uribe, "Beyond the Navier-Stokes equations: Burnett hydrodynamics," Phys. Rep. 465, 149-189 (2008).

[47] C. Cercignani, Mathematical Methods in Kinetic Theory (Plenum Publishing Inc., 223 Spring Street, New York, N.Y. 10013, 1990).

[48] L. J. Klinkenberg, "The permeability of porous media to liquid and gases," in API 11th Mid Year Meeting (API Drilling and Production Practice, Tulsa, Oklahoma, 1941) pp. 200-213.

[49] G. E. Karniadakis and A. Beskok, Micro-flows, Fundamentals and Simulation (Springer-Verlag, New York, 2002).

[50] K. Suga, S. Takenaka, T. Ito, M. Kaneda, T. Kinjo, and S. Hyodo, "Evaluation of a lattice Boltzmann method in a complex nanoflow," Phys. Rev. E 82, 016701 (2010).

[51] M. Barisik and A. Beskok, "Scale effects in gas nano flows," Phys. Fluids 26, 052003 (2014). 